\title{
Applications of Blockchain Technology in Clinical Trials: Review and Open Challenges
}

This paper was downloaded from TechRxiv (https://www.techrxiv.org).

\section{LICENSE}

CC BY-NC-SA 4.0

SUBMISSION DATE / POSTED DATE

09-07-2020 / 15-07-2020

\section{CITATION}

Omar, Ilhaam; Jayaraman, Raja; Salah, Khaled; Yaqoob, Ibrar; Ellahham, Samer (2020): Applications of Blockchain Technology in Clinical Trials: Review and Open Challenges. TechRxiv. Preprint. https://doi.org/10.36227/techrxiv.12635783.v1

DOI

10.36227/techrxiv.12635783.v1 


\title{
Applications of Blockchain Technology in Clinical Trials: Review and Open Challenges
}

\author{
Ilhaam A. Omar ${ }^{1}$, Raja Jayaraman ${ }^{1}$, Khaled Salah², Ibrar Yaqoob², Samer Ellahham ${ }^{3}$ \\ ${ }^{1}$ Department of Industrial \& Systems Engineering, Khalifa University, Abu Dhabi, UAE. \\ ${ }^{2}$ Department of Electrical Engineering and Computer Science, Khalifa University, Abu Dhabi, UAE. \\ ${ }^{3}$ Heart \& Vascular Institute, Cleveland Clinic Abu Dhabi, Abu Dhabi, UAE. \\ ilhaam.omar@ku.ac.ae,raja.jayaraman@ku.ac.ae, Khaled.salah@ku.ac.ae,ibrar.yaqoob@ku.ac.ae, \\ EllahaS@ClevelandClinicAbuDhabi.ae
}

\begin{abstract}
Blockchain technology has disclosed unprecedented opportunities in the healthcare sector by unlocking the true value of interoperability. Specifically, the striking features of blockchain technology, such as data provenance, transparency, decentralized transaction validation, and immutability can help to compensate for stringent data management issues (e.g., patient recruitment, persistent monitoring, data management, and data analytics and accurate reporting) in clinical trials (CTs). Although several research studies show that blockchain solutions help to improve patient retention, data integrity, privacy, and ensure CTs compliance with regulatory policies, a comprehensive survey on this topic is lacking. In this survey, we provide insights into the adoption of blockchain technology in CTs. We categorize and classify the literature by devising a meticulous taxonomy of the decentralized tasks of CT and practices based on indispensable parameters. Furthermore, we provide insights on works in progress towards deploying blockchain solutions in CTs. Finally, we identify and discuss several challenges that hinder the successful implementation of blockchain technologies in CTs.
\end{abstract}

Keywords: Blockchain; smart contracts; Ethereum; data management; clinical trials; healthcare

\section{Introduction}

The extraordinary proliferation of cryptocurrencies has added notable contributions in the financial sector because they do not require any third party, thereby holding an intrinsic value. Nevertheless, the success of cryptocurrency depends on the implementation of blockchain technology, which provides several advantages, such as auditability, decentralization, anonymity, persistency, among others. Evidently, blockchain technology has a wide range of applications, e.g., financial, supply chain, engineering maintenance, healthcare management, and the Internet of things (IoT) [2]. Specifically, the convergence of blockchain with IoT can significantly improve transaction processing in the telecommunication industries, as it serves as a distributed ledger that keeps track of all transactions through a consensus algorithm across a computer network. The consensus algorithm validates all the peer-to-peer transactions without any intervention of a centralized authority, wherein each valid transaction is timestamped and digitally signed by the sender, thereby generating a cryptographically reliable proof of its provenance. Subsequently, all the validated transactions are added into the blockchain as a new "block" that cannot be modified or removed. Thus, blockchain technology is suitable for such applications where collaboration between multiple participants and data transparency are equally important in the network.

Even though blockchain has gained immense popularity in the financial sector, it is gradually gaining attention in other sectors, especially in healthcare. For example, a blockchain company named Factom [3] is closely working with HealthNautica [4] to utilize the bitcoin blockchain technology to aid in storing and auditing of medical records. Another company named Gem [5] is working with Capital One to efficiently reduce the processing time while filing health insurance claims. Similarly, DNA. Bits [6] aims to employ blockchain technology for storing medical and genetic data as a personalized "DNA wallet". BlockVerify [7] uses this technology to fight against counterfeit drugs by tracking the unique verification tag placed on drug packages to verify its authenticity and provenance against the blockchain. Based on the aforementioned case studies, it is evident that the inherent features of blockchain can significantly enhance the healthcare and biomedical sector [8]. 
To further emphasize the need and importance of blockchain technology, evidence shows that trust is always a question in medical research as data can be manipulated. Since drug developers are always looking forward to seeing positive results in order to get approval and release their new drug out in the market [9]. Consequently, clinical trials (CTs) must test the effectiveness of new drugs, highlight side effects, and also be able to compare their advantages and limitations before their release. On the other hand, data integrity is an important issue because data obtained from trials aren't instantly made public to stakeholders, such as doctors, researchers, and patients, resulting in a trustless environment, thereby transparency is crucial. In 2007, the United States Food and Drug Administration (FDA) has attempted to impose some order that all CTs must be registered in a public database named ClinicalTrials.gov [10]. However, a recent study shows that more than half of the trials registered have failed to publicize their results [11]. Therefore, implementing a blockchain-based system can reinforce trust, and radically minimizes the falsification of published data. Also, it is worth noting that as CTs can become more transparent and trustworthy, the analysis of collected data becomes more accurate, thereby increasing the capability of saving more lives. Hence, utilizing blockchain technology to manage CT databases shows great potential for enhancing research in pharmaceutical industries. In addition, blockchain has the capability to disrupt the CT industry while diminishing the use of legacy data management platforms. IBM reveals that $56 \%$ of healthcare executives have plans to implement commercial blockchain solutions by 2020 [13]. Moreover, CoinDesk reports that three big pharmaceutical companies Sanofi, Pfizer, and Amgen are working together towards implementing blockchain solutions to speed up the process of testing new drugs, which aids as a big boost for evidence-based CT [14]. Blockchain technology is in its infant stage. The researchers are still performing experiments to measure its impact in CTs for resolving certain challenges, such as patient privacy and enrollment, regulatory approval, and data integrity and reproducibility. Although a few studies have proposed consensus algorithms and possible architectures suitable to meet the needs of CTs, the current literature lacks a comprehensive feasibility study on the adoption of blockchain in CTs.

The contributions of the study are as follows:

- We outline the vision for applying blockchain solutions in CTs by highlighting their distinctive features and benefits.

- We taxonomize the recent literature to interpret the role of blockchain in CTs in the context of CT tasks and practices, blockchain type, and consensus protocol.

- We provide insights on works in progress towards deploying blockchain solutions in CTs.

- We identify, enumerate, and discuss several indispensable challenges involved in the implementation of blockchain solutions for CTs.

These contributions are provided in separate sections from II to V. Section VI provides concluding remarks.

\section{Background}

This section gives an overview of blockchain technology and CTs. It also discusses the key areas in CTs, whereby blockchain technology can help to perform transformation and enhancement.

\section{A. Blockchain}

Blockchain technology has a vast range of capabilities that are worth exploring as it not only enhances existing applications but also gives room for new applications to emerge and develop. This innovation initially originated from a combination of different domains including cryptographic science, distributed consensus algorithm, software engineering, mathematics, and economic game theory [15, 16, 17]. Essentially, the blockchain is a distributed ledger, where all the validated transactions are stored as a chain of blocks. The chain continues to grow as new transactions are built and blocks are added into it. The blockchain platform is distinguished by its key characteristics enabling it to be a promising disruptive technology that reduces the emphasis on traditional data management systems. These distinct advantages of blockchain are shown in figure 1, where their explanations can be found in $[18,19,20,21]$. 


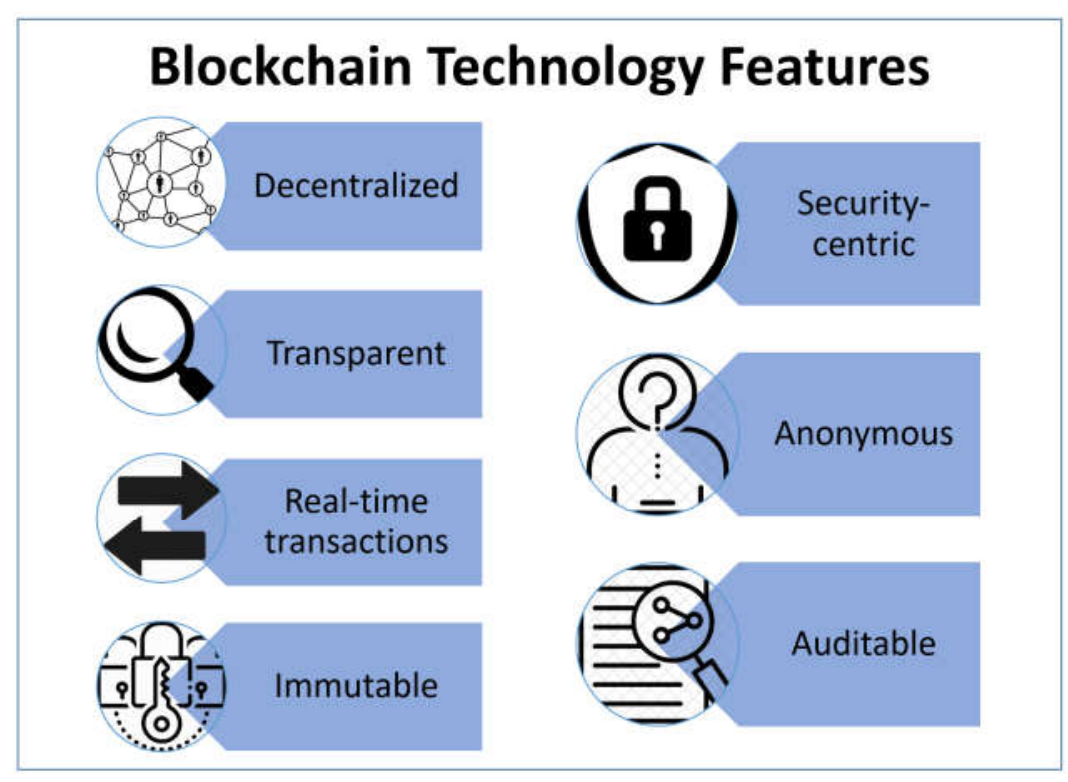

Figure 1: Key elements in a blockchain

Blockchain can be applied to an application, where transactions occur and assets are managed. This is made possible because of smart contracts. A smart contract is a set of rules written in the form of scripts that can be embedded within the blockchain to administer a transaction. This digital contract is automatically executed before a transaction commits, hence, acting as a smart software agent [15, 22]. Recent blockchain platforms such as the Ethereum platform is designed to support smart contracts. The Ethereum protocol was built to increase functionality and allow flexibility in programming of different types of smart contracts within Ethereum. These contracts are supported using Ethereum's cryptocurrency called Ether so that users can build various contracts, applications or even services [23].

Currently, data storage is expensive and limited in blockchain. Therefore, decentralized storage mechanisms are potential solutions for blockchain-based companies to explore and implement. The decentralized storage systems provide an ability to store files without the interference of a central party. An example of this decentralized storage technology is the InterPlanetary File System (IPFS) [24]. In this system, a unique cryptographic hash is given to each file that is stored on the IPFS network, thereby making the history of each file immutable and traceable. Moreover, in this system, the downloading speed is faster as the files are distributed over the network. Thus, these features make IPFS as an ideal place to store data because it uses time-stamped through blockchain technology. As a result, IPFS links stored in the blockchain are directly referenced to the information stored on the IPFS network without storing the actual data on the blockchain itself. Hence, a combination of these two technologies is beneficial as IPFS can be used in blockchain applications that require a decentralized database where its authenticity can be verified. Other examples of this decentralized storage technology include FileCoin [25] and Swam [26].

\section{B. Clinical Trials}

New drugs and treatments are tested in various phases of a CT to find ways to counteract recently discovered or existing pathologies. These phases in a CT serve as steps that scientists take to experiment with new drugs or treatments on a selected group of patients to validate its efficacy. The trials that involve new drugs, in particular, are commonly conducted in four phases as shown in figure 2. In phase I, a few patients participate for testing purposes, later on, the number of participant increases as the drug moves to the next phase to test the safety and effectiveness of the drugs. Therefore, it is important that this CT process is carried out ethically in order to protect the wellbeing of participating patients. Moreover, the recorded data and updated documents must be tracked continuously in order to ensure that the trial is aligning with the regulatory requirements while assuring that the reported clinical study findings are reliable. 


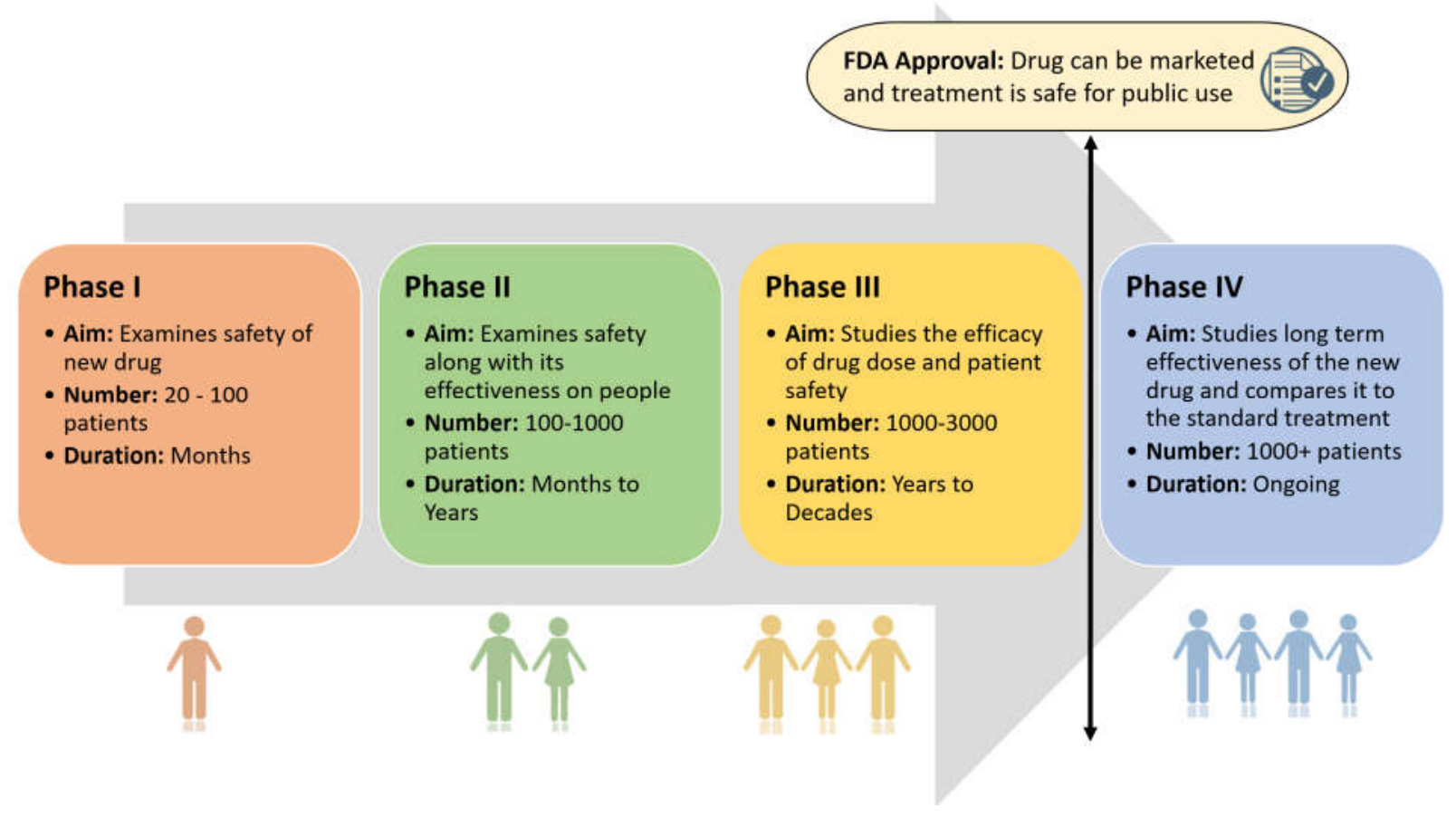

Figure 2: Different phases in CTs

A general CT process flow in a drug testing experiment is broadly summarised in figure 3 . Initially, each trial requires a comprehensive protocol that must be approved by the regulatory authorities. Subsequently, this protocol is implemented and a selected group of patients are chosen based on the inclusion and exclusion criteria [27]. Later on, these patients provide consent forms and randomized into two groups, the treatment, and control or placebo groups. The former group is subjected to the proposed treatment while the latter acts as a control group that is given a placebo instead. The progress of these patients is tracked and recorded as raw data. The collected raw data can be in various forms including surveys and interviews, laboratory tests, and medical examinations. Finally, this data is statistically analyzed and evaluated to show improvements in outcomes and justify the results obtained and submitted to the regulatory authorities for approval. Thus, it is clear that data accuracy and integrity are substantial in CTs as they must comply with the regulations outlined by regulatory authorities such as the Good Clinical Practice (GCP) guidelines set by the US FDA [27]. As a result, developing a reliable and trustworthy system to oversee the process still remains a challenge when carrying out CTs.

The stakeholders involved in a CT system are FDA, Institutional Review Board (IRB), Sponsor, Contract Research Organization (CRO), Principal Investigator (PI), physicians, and patients. Figure 4 presents the general information flow of these stakeholders under the existing setup. It can be seen that the CRO plays a vital role in CTs as the clinical study closeout report is generated and verified by them and later send to the sponsor for reporting it to the FDA and IRB. Table 1 summarizes the roles and key responsibilities of these stakeholders [28, 29]. The current CT system is centralized around the sponsor and the process is monitored and controlled by the CRO. 


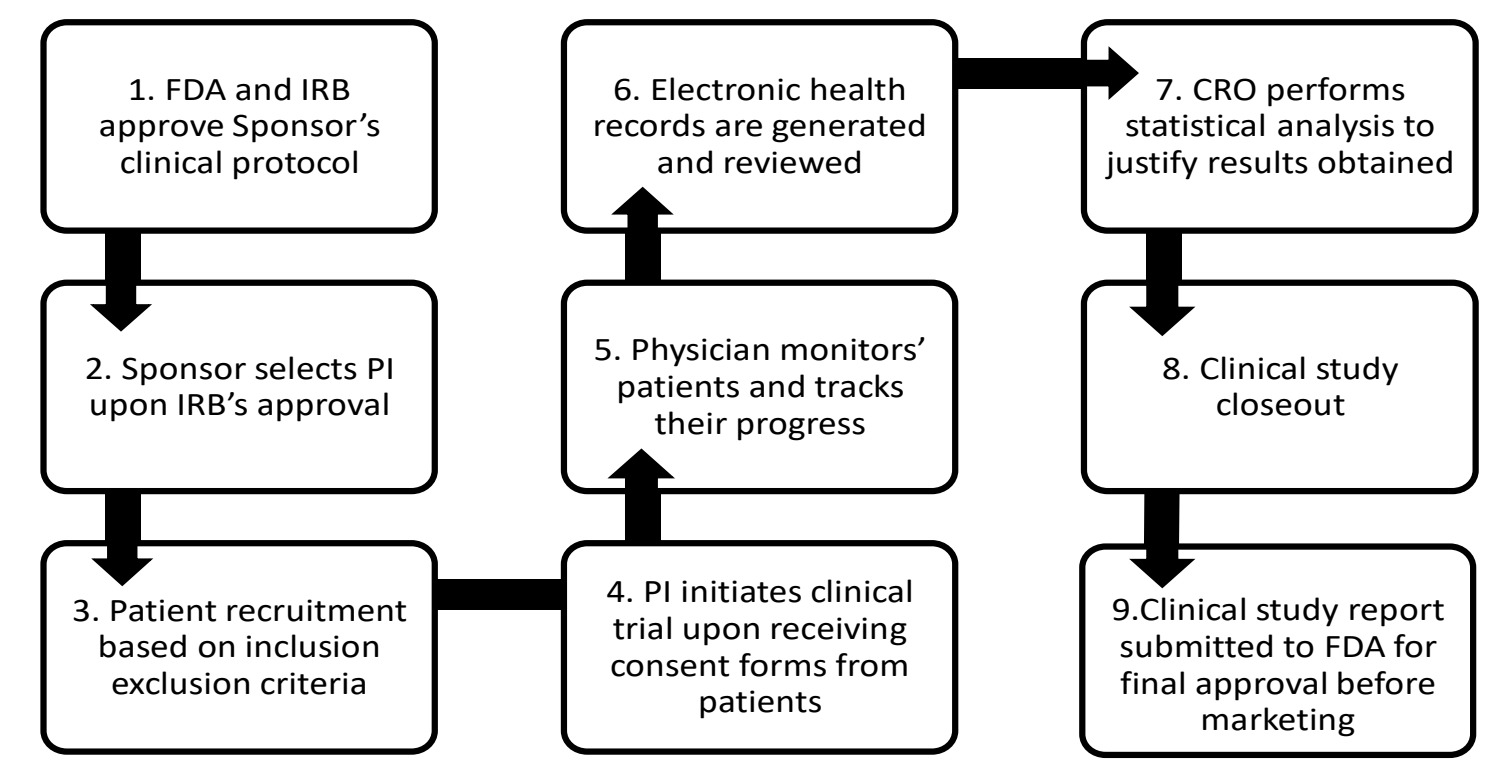

Figure 3: Process sequence of CT Systems

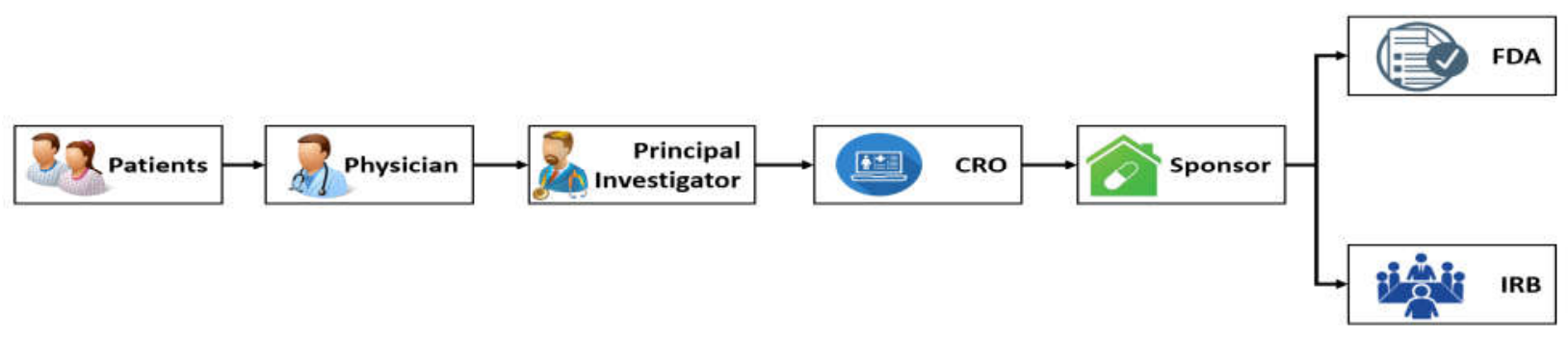

Figure 4: Sequential information flow of stakeholders under the existing setup

Table 1: Responsibilities of stakeholders in a CT

\begin{tabular}{|c|c|}
\hline Stakeholder & Key Responsibilities \\
\hline FDA & $\begin{array}{l}\text { - To implement a regulatory system that all CTs must follow. } \\
\text { - To ensure drugs available in the country to fulfill the necessary requirements for safety, } \\
\text { quality, and efficacy. } \\
\text { - To close down an on-going trial in case if there are serious breaches of GCP }\end{array}$ \\
\hline IRB & $\begin{array}{ll} & \text { To supervise and monitor each step of the trial. } \\
\text { - } & \text { To safeguard the welfare and rights of participants. }\end{array}$ \\
\hline Sponsor & $\begin{array}{ll}\text { - } & \text { Appointing competent investigators. } \\
\text { - } & \text { Shipping all drugs necessary for a trial. } \\
\text { - } & \text { To file all required documents to the FDA. } \\
\end{array}$ \\
\hline $\mathrm{CRO}$ & $\begin{array}{ll}\text { - } & \text { Study management and administration. } \\
\text { - } & \text { Site qualification, selection, and initiation. } \\
\text { - } & \text { Database management. }\end{array}$ \\
\hline PI & $\begin{array}{l}\text { - To develop the concept for the trial. } \\
\text { - } \quad \text { To write and submit the CT protocol for IRB approval. } \\
\text { - } \quad \text { Recruiting patients and managing the informed consent process. }\end{array}$ \\
\hline Physician & $\begin{array}{l}\text { - To treat patients according to the CT protocol. } \\
\text { - To review and interpret lab results, ECGs, adverse events, among others. } \\
\text { - To keep records of how each patient responds to the treatment and reports any side } \\
\text { effects encountered. }\end{array}$ \\
\hline Patient & $\begin{array}{l}\text { - To provide the informed consent form. } \\
\text { - To understand the role of IRB in terms of protecting patient wellbeing. }\end{array}$ \\
\hline
\end{tabular}




\section{How Blockchain Can Transform Clinical Trials}

A study has reported that almost $80 \%$ of the medical studies are non-reproducible [30] due to the presence of various errors, such as fraud, data misrepresentation, and trial misconduct. The enhancement of the research quality not only ensures improved reproducibility but also empowers the research communities with a secured network for sharing data. Blockchain technology can be employed to compensate for patients' identity and privacy issues. The technology provides a decentralized data tracking system that reinforces trust between different stakeholders involved in a CT. Additionally, it ensures transparency between patients and physicians as well. Blockchain technology can set up a strong foundation towards an enriched clinical study practice as discussed in [31]:

1. Blockchain offers data traceability features that can help to improve the safety of drugs throughout the life cycle of a CT.

2. Guaranteeing the clinical protocol complies with the GCP and regulatory code of conduct through data security and verification.

3. Increasing competitiveness through better transparency, accessibility, and trackability.

Furthermore, this technology can help to drastically transform potential areas of CTs, as shown in figure 5 .

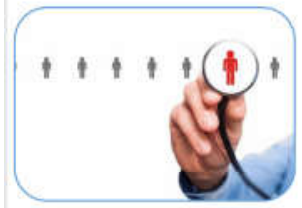

Patient recruitment

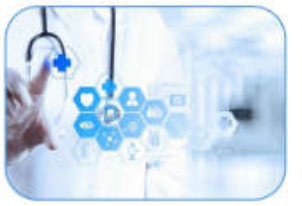

Medical data sharing and privacy

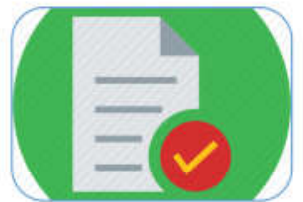

Data integrity

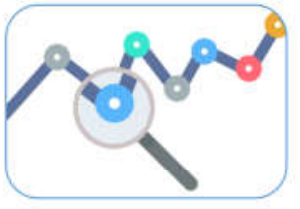

Consent traceability

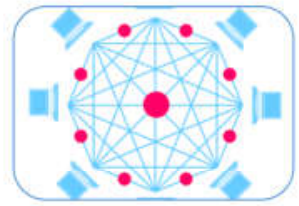

Transparency

Figure 5: Potential CT areas transformed via blockchain implementation

\section{- Patient Recruitment}

Patient recruitment is considered to be one of the most laborious procedures in the CTs process. It has been estimated that it takes up almost $30 \%$ of the clinical timeline [32] where some clinical sites even fail to enroll a single patient. Blockchain distributed ledgers have the potential of reducing this time significantly as they help to connect patients' registered and trial sites anonymously. Subsequently, the PI can select potential participants from a pool of patients according to the inclusion and exclusion criteria defined in a protocol.

\section{- Medical Data Sharing and Privacy}

Approximately half of the conducted trials fail to produce desired results. The Stat News has reported that almost $90 \%$ of the trials registered in "ClinicalTrials.gov" lack study results [33]. This raises a concern of safety for patients and creates a knowledge gap for potential drug investigators. Thus, adopting a blockchain solution can provide a secure tracking system as the ledger contains all the updated information, thereby allowing all participants to know the current status of the $\mathrm{CT}$, which is being conducted. Also, protecting the identity of patients can be easier as each patient has an encrypted address which is mapped to his/her identity, where their information can be accessed only in two ways. Either the patients agree to provide their private keys to the designated trial site or the appointed physician creates and holds the patient's keys throughout the CT process in order to monitor their response with regards to the drug treatment.

\section{- Data Integrity}

Data recorded in CTs are expected to be genuine and accurate. Data integrity in the blockchain is assured by validating each transaction using a consensus algorithm. In case of fault or error detection in a chain, it is possible to trace back and identify the origin of the problem along with the person responsible for entering this information [19]. As a result, recorded CT data in the blockchain can be a trusted source in clinical research. 


\section{- Consent Traceability}

Obtaining patient consent is a challenging yet essential part of GCP. It has been stated that almost $10 \%$ of the clinical studies have problems directly linking to patients' consent [34]. This can be in various forms such as invalid or unapproved consent forms and failure to obtain re-consent forms in certain conditions where the clinical protocol is revised or updated. Current proof of concept experiment conducted by researchers showed that the blockchain was able to trace and link each timestamped patient consent to the right version of the revised CT protocol when cryptographic validation was applied on each transaction [30, 35].

\section{- Transparency}

Using blockchain technology to track CTs throughout their lifespan is not only restore trust in the results obtained, but also encourages more people to participate. Transparency is a very important parameter when new drugs are investigated as it reduces the probability of clinical sites that are selectively reporting optimistic results [9]. It should be noted that if trials are timestamped and recorded at the time of establishment, then this increases the chances of results to be reported as noisy data instead of meaningful data. Hence, the implementation of blockchain technologies in clinical research does not only ensure transparency and data integrity but also promotes collaboration between different clinical sites and stakeholders. Regulatory agencies and healthcare companies are looking forward to seizing the opportunities brought about by this technology. It was reported in 2017 by the Healthcare IT News that the Centers for Disease Control and Prevention along with the FDA have agreed to collaborate with IBM Watson Health in running pilot blockchain projects to explore its real-world benefits in database management and data sharing [36]. Table 2 summarizes the literature available on the convergence of blockchain and CTs.

Table 2: Recent literature on applications of blockchain in CTs

\begin{tabular}{|c|c|c|}
\hline Reference & Research Objective & Proposed Approach \\
\hline [59] & $\begin{array}{l}\text { - To prevent data fabrication done by } \\
\text { researchers and pharmaceutical } \\
\text { companies to achieve data integrity. }\end{array}$ & $\begin{array}{l}\text { Developed a Bitcoin blockchain-based } \\
\text { system to validate ongoing CTs by producing } \\
\text { a proof of concept based on a research } \\
\text { protocol available on the ClinicalTrials.gov } \\
\text { website. }\end{array}$ \\
\hline [27] & $\begin{array}{l}\text { To provide a legal and ethical } \\
\text { framework for auditors to validate } \\
\text { CTs. }\end{array}$ & $\begin{array}{l}\text { Proposed a blockchain-based provenance } \\
\text { system named Scrybe that uses a novel } \\
\text { lightweight mining algorithm instead of the } \\
\text { PoW consensus algorithm. }\end{array}$ \\
\hline [60] & $\begin{array}{l}\text { To propose } \mathrm{s} \text { a framework to securely } \\
\text { capture and verify provenance data. }\end{array}$ & $\begin{array}{l}\text { Proposed a system called DataProv which } \\
\text { combines blockchain with cryptographic } \\
\text { techniques to ensure data provenance. }\end{array}$ \\
\hline [61] & $\begin{array}{l}\text { To provide an easier way to share and } \\
\text { exchange healthcare records to view } \\
\text { the progress of a CT. }\end{array}$ & $\begin{array}{l}\text { Proposed a blockchain-based system called } \\
\text { BlocHIE for healthcare information } \\
\text { exchange. }\end{array}$ \\
\hline [62] & $\begin{array}{l}\text { To find an easier method to share data } \\
\text { in order to enhance the decision- } \\
\text { making process. }\end{array}$ & $\begin{array}{l}\text { - Proposed FHIRChain, which is a blockchain- } \\
\text { based architecture used to share clinical data. }\end{array}$ \\
\hline [63] & $\begin{array}{l}\text { To reduce the cost of conducting } \\
\text { multi-site trials while ensuring data } \\
\text { integrity and patient privacy. }\end{array}$ & $\begin{array}{l}\text { Proposed a data management framework } \\
\text { based on private blockchain technology and } \\
\text { smart contracts. }\end{array}$ \\
\hline [64] & $\begin{array}{l}\text { To focus on the workflow of health } \\
\text { information Exchange and CTs in } \\
\text { order to identify the means by which a } \\
\text { private blockchain can tackle serious } \\
\text { issues. }\end{array}$ & $\begin{array}{l}\text { Introduced a proof-of-concept for potential } \\
\text { solutions to monitor CTs across different } \\
\text { regions. }\end{array}$ \\
\hline [65] & $\begin{array}{l}\text { To highlight the role of blockchain in } \\
\text { terms of data management of CTs. }\end{array}$ & $\begin{array}{l}\text { - Developed a proof-of-concept } \\
\text { implementation of a patient-facing and } \\
\text { researcher-facing system. }\end{array}$ \\
\hline
\end{tabular}




\section{Taxonomy of Blockchain for Clinical Trials}

This section explains the key concepts that enable blockchain technologies to be beneficial when applied to CTs. Figure 6 categorizes and classifies these concepts as a tree structure of existing literature. They are classified primarily as decentralized CT tasks and practices, available blockchain types and consensus protocols that can be used for decentralized transaction verification across the blockchain network.

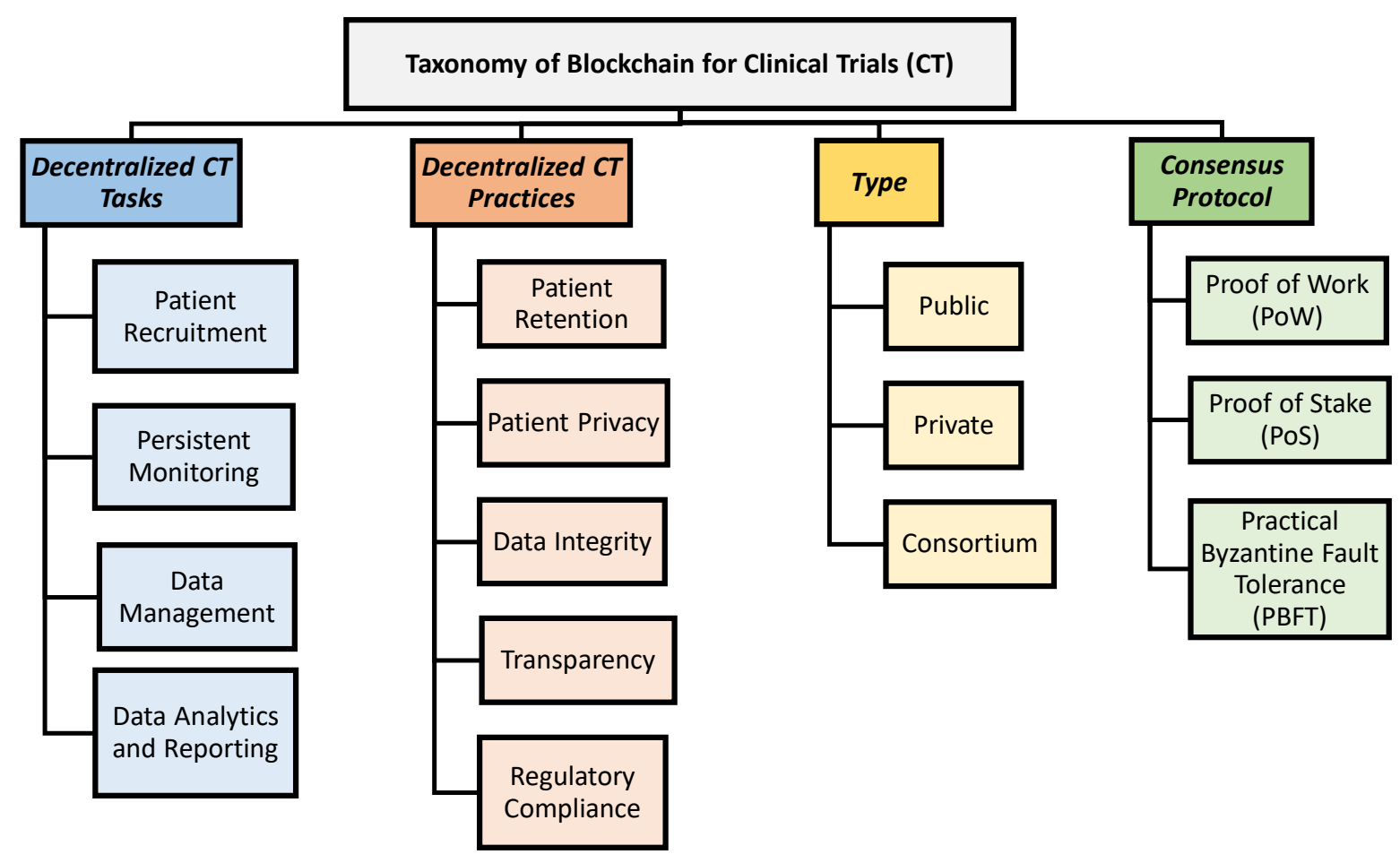

Figure 6: Taxonomy of Blockchain for Clinical Trials

\section{A. Decentralized Clinical Trial Tasks}

Herein, we envisage how blockchain implementation helps to validate the authenticity of transactions.

\section{- $\quad$ Patient Recruitment}

A recent study has reported that an approximately $\$ 2.6$ billion amount is spent on developing new drugs right from its manufacturing to the marketing stage [37]. A major chunk of this cost is spent on recruiting patients and finding suitable means to retain them throughout the CT process. Some of the trials need more than thousand patients where each must satisfy the criteria defined in the protocol. It has stated that $80 \%$ of the clinical studies are postponed because of recruitment issues [38]. Hence, delayed recruitment period extends the lifetime of the trial which consequently delays the chances of new drugs to be available in the market, thereby causing patients to wait for years until new treatment opportunities are made available.

The blockchain technology can aid in two important CT recruitment tasks. First, it enables sponsors to efficiently recruit the right sample of patients required, and second, it increases patients' awareness regarding new trials being conducted by matching the patient's profile to the protocol's inclusion and exclusion criteria [39]. The broadcasting of CT information to targeted clinical sites can be achieved using the smart contract feature available in the Ethereum platform [22]. In addition, the smart contract can be programmed to automatically validate the legitimacy of sponsors, clinical sites and verify the qualification of participating patients. As a result, Ethereum blockchain can be used to recruit patients where all sponsors and clinical sites have their own accounts registered in the CT smart contract. This smart contract is made unique for each new trial broadcast. It also makes sure that an Investigational New Drug (IND) approval from the FDA is received before the trial moves on to the recruiting phase. This current blockchain technology (Ethereum) strengthens trust among different parties including sponsors, clinical sites and patients, thereby guaranteeing that trial standards and patient safety are not compromised [40]. 
Another proposed solution shows that significant results in the digital health sector can be obtained by merging IoT and blockchain technologies [38] [41]. The IoT devices enable patients to generate a massive amount of data that can be uploaded to clinical sites that help in prescreening the potential participants according to defined inclusion and exclusion criteria. It also helps to predict whether or not sufficient patients are available that match the criteria required in a specific population. Thus, the convergence of IoT with blockchain can provide an appropriate environment where patients' confidentiality is assured.

\section{- $\quad$ Persistent Monitoring}

In traditional CT systems, patients communicate only with clinical sites where their conditions are recorded using a questionnaire or diary. However, with the help of blockchain technology, this information is made available to all members reside in the network as it is a distributed decentralized ledger, which helps the FDA and sponsors to constantly monitor the captured data. The FDA has a right to audit any transaction in the blockchain and also review the reports uploaded directly by physicians rather than waiting to receive them from sponsors.

Persistent monitoring can be ensured using a private blockchain where clinical sites, sponsors, FDA and IRB act as active participants in this closed network. Miners in a network validate the transactions and they are distributed in such a way that they can avoid 51\% attacks [42]. CT contracts are designed to accommodate the resource contribution of miners. All participants are able to audit the ledger and any attempt of false entry into this ledger can be viewed by all users in the network. This is beneficial as raw data can be seen by the FDA in a real-time without passing through intermediaries. Also, it reduces the chances of data fabrication and provides an immutable chain of recorded transactions.

\section{- Data Management}

The patient's data gathered during the CT phase are quantified and analyzed by the CRO using statistical tools. At the end of the trial, a final report is generated and handed over to the sponsor to present the finalized CT outcomes to the FDA that help to obtain a new drug approval. Thus, the FDA's judgment is solely based on the clinical data presented to them. For this reason, it is vital that the CT staff adhere to the standards outlined in the CT protocol. Thus, Clinical Data Management (CDM) plays an essential role in CTs as it is responsible for the collection and verification of patients' information [43]. Its prime objective is to record high-quality data while identifying any missing information that is needed for analysis to accelerate the CT management process. Only high-quality data are qualified for statistical quantification. There are several software systems available that support clinical data management (CDM) in several stages of the CT. The study conducted in [43] summarizes these systems in a tabular form according to the data management stage and its applications. These systems were developed to support large complex trials.

The current studies, for example [44] has channeled their focus towards blockchain as a mean of improving CDM. Examples of such projects include Gem Health Network [45], Factom [3], BitHealth [46], MedVault [47] and Healthcare Data Gateways [48]. Moreover, companies such as Accenture [49] and Deloitte [50] have also started investigating this technology in storing and managing healthcare records. Another company called Guardtime had partnered with the Estonia government to create a blockchain-based system that secures medical records and verifies patient identities $[44,51,52]$.

\section{- Data Analytics}

The integration of blockchain technology with machine learning and artificial intelligence can help to perform data analytics in the healthcare sector [53]. By doing so, the data analysis process is carried out automatically without any intervention of sponsors or clinical sites. Data analytics tools are used to generate statistical reports which are uploaded on the blockchain network for all parties to view. This allows the FDA to check reports of any serious adverse event (SAE) in a real-time manner. The current CDM systems require clinical sites to manually type the results; however, blockchain automates this process by having an analytics node in the network which is in-charge for data cleaning and detecting any falsified data entry. 


\section{B. Decentralized Clinical Trial Practices}

The decentralized CT practices lead to providing a standard mode of retaining patients, maintaining patient confidentiality, guaranteeing data integrity and transparency while ensuring that CTs compliance with GCP guidelines. The following subsection explains how blockchain technology helps to decentralize such practices.

\section{- Patient Retention}

In the years 2005-2016, around 10,000 new research and development partnerships were created by Life Science companies with the objective of encouraging scientific discoveries [31]. These partnerships focused on improving the rate of patient retention at CTs. It has been reported that almost $85 \%$ of CTs fail to retain their patients, as roughly $30 \%$ of them tend to drop out of these trials [31]. This increased dropout rate is mainly because patients are reluctant to visit trusted clinical sites that are usually located far away from them. Blockchain technology enables the sponsor to use more clinical sites for testing patients' progress while assuring that the collected raw data are genuine. Hence, this encourages more patients to participate in CTs, as it is easily accessible and convenient for them. In short, implementing blockchain solutions to CTs encourages patients to select clinical sites that are more convenient to them, thereby improving patient retention throughout the lifetime of CTs.

\section{- Patient Privacy}

During the past decade, data crime has become very common in the healthcare sector because multiple stakeholders are usually responsible for monitoring and handling patient records despite the fact that current CDM software systems lack security measures to avoid data breaches [31]. For instance, it has been reported that in 2018, 351 data breaches of more than 500 healthcare records were resulted in exposing 13,020,821 medical records [54].

The main features of blockchain are its enhanced privacy and security that are made possible through cryptographic algorithms. For example, the Bitcoin blockchain uses the cryptographic hash function called 256bit Secure Hash Algorithm (SHA-256). The SHA-256 function runs the proof-of-work (PoW) algorithm and also enhances user privacy, as each user is given a hash value in replace of the IP address; since the IP can be easily used to expose their true identity [44]. Moreover, Bitcoin blockchain utilizes the 256-bit Elliptic Curve Digital Signature Algorithm which is an asymmetric cryptography algorithm, which is specifically designed to ensure digital asset ownership, such as medical records through the use of digital signatures which are created and validated using public and private keys [44].

\section{- Data Integrity}

In most cases, physicians, medical laboratories or even sponsors usually make data entry errors and responsible for data falsification. Therefore, guaranteeing data accuracy remains taxing for the FDA as they merely receive aggregated reports from sponsors after each trial, thereby the approval process is time-consuming because the current CT system makes it difficult to carry out timely corrections of missing or wrong data. Furthermore, the FDA lacks the manpower required to frequently audit every single registered CT application. In this context, implementing a blockchain system can enable the FDA to become an active member during the CT process as this system ensures data provenance and accuracy and also possesses analytic capabilities to perform statistical analysis on recorded data.

\section{- Transparency}

The World Health Organization has demanded that all the methodologies and results of CTs made accessible for everyone to view. However, a recent study reveals that almost half of the registered trials have failed to publicize their findings [55]. Hence, there is a great need for transparency. Blockchain-based approaches can be used to achieve transparency in the healthcare sector, wherein smart contracts act as a trusted CT administrator. A study conducted in [55] proposes the utilization of a private Ethereum blockchain network because its protocol supports smart contracts. These contracts track all changes made to sensitive data, such as CT protocol, and patient consent and their progress.

\section{- Regulatory Compliance}

The guidelines set by regulatory authorities, such as FDA can be seen as a set of business rules that can be programmed into the blockchain networks [19]. Current systems that are used to validate and audit recorded data 
are labor-intensive and expensive. These limitations can be overcome by employing the blockchain technology as it significantly helps to unburden the load. Since, different actors are involved in the CT, blockchain-based solution can help to validate data and the protocol as data stored in the blockchain is immutable and every step in the protocol can be validated through the use of smart contracts.

\section{Blockchain Types for Clinical Trials}

There are mainly three types of blockchains, namely public, private, and consortium that can be used in CTs research. The former is an open-source network that allows anyone to participate in the network. This type of network typically has an incentive mechanism in order to encourage more participants to join. On the other hand, the latter places a restriction on who is allowed to enter and participate in the network, once anyone joins the network, the blockchain continues to work in a decentralized manner. Consortium blockchains are jointly controlled by a group of organizations. Further details are provided in the next subsection.

\section{- Public, Private, and Consortium}

Public blockchains are of permissionless type, where there is no need for intermediaries and anyone can join the network to perform read and write operations on CT data. In public blockchains, all transactions can be checked and verified by anyone and their consensus mechanisms are also open for everyone to participate [18]. Bitcoin and Ethereum are well-known examples of the public blockchain. The core advantage of such type of blockchain is security as it is open for everyone so it will be harder for any malicious entity to gain control over the network where CT data is stored. Moreover, in public blockchains, all the data is open to the public for verification, thereby ensuring transparency. Some of the limitations of public blockchains include extremely slow transaction processing time, scalability, and energy consumption.

Private blockchains are known as permissioned blockchains. They do not allow everyone to join the network. They control reading and writing operations into the blockchain. Such type of blockchain is mainly used by financial or healthcare organizations to ensure efficiency and auditability [21]. Furthermore, mining is not necessary in the private blockchain, as a single private entity has sole ownership of creating and verifying blocks [15]. Hyperledger fabric is one of the common examples of private blockchains [56]. Private blockchains can help to solve three fundamental problems related to CT data, such as data privacy, compliance, and cost and speed. Unlike the public blockchain, only authorized or designated organizations/entities can join the network wherein CT data is stored, thereby ensuring data privacy. Private blockchains can easily achieve data privacy and security compliance (i.e., HIPAA and PIPEDA) because CT data is stored on authorized nodes. Public blockchain ledger usually requires high computation power which can make it expensive for CT data management. However, in permissioned blockchains, one can utilize computationally inexpensive protocols to verify CT data related transactions.

The consortium is known as hybrid or partially private blockchain. In such type of blockchain, ledger is distributed only to permitted participants while the consensus mechanism is controlled by selected servers using rules that are agreed upon by all participants in the network. As a result, the network is partly decentralized. Consortium blockchain platforms almost have the same advantages as a private blockchain. However, they operate under different groups instead of a single entity. The major advantages of consortium blockchain platforms are that their consensus mechanism consumes less energy, and this platform is more flexible and ensures greater privacy compared to the public blockchain. Although consortium blockchain platforms are under the control of certain organizations, they are protected from monopoly. One of the key issues posed by consortium blockchain is that it is vulnerable to malicious players due to its centralized structure.

\section{The Role of Consensus Protocols in Clinical Trials}

This subsection presents the common consensus protocols and explains how blockchain can exert considerable impact on the performance of CTs.

\section{- Proof of Work}

The Proof-of-Work (PoW) consensus algorithm has been used in Bitcoin networks [1]. The PoW adds a new block into the existing blockchain network through solving a complex mathematical problem. The nodes that are 
engaged in solving this problem are called miners; whereas, the PoW mechanism is called mining [21]. This PoW model solves the double-spending problem and ensures security, thereby preventing any fraudulent activity. Another advantage of the PoW is that it is highly scalable which makes it suitable for numerous applications. However, one of the limitations of $\mathrm{PoW}$ is that it requires intensive computing power to validate the transactions that makes it very expensive. Additionally, it is vulnerable to $51 \%$ attacks [57].

\section{- Proof-of-Stake}

Proof-of-stake (PoS) was introduced to overcome the shortcomings of PoW, especially in terms of high energy consumption. Ethereum is currently working towards switching to a PoS model which aims to transform the reward system. Miners are no longer required in a PoS model as they are replaced with validators. Cryptographic challenges would no longer be used because validators are required to put their own ether (Ethereum's cryptocurrency) at stake to validate a block. This means in the case of any malicious activity, the validators lose their ether. The reward system enables the validators to earn a transaction fee upon validating each smart contract and transaction [18]. Therefore, making it more energy-efficient, as it encourages collaboration over the competition because the sooner everyone agrees on a transaction, the faster the blocks would be created, leading to higher profit generation [21]. In addition, people would also have to pay a gas price or a fee if they would wish for their transaction or smart contract to be executed and added to the blockchain. The key advantages of PoS are higher transaction processing speed compared to PoW, less energy consumption, and fewer hardware requirements. Despite many advantages, $\mathrm{PoS}$ is still vulnerable because only the richest person can have control over the consensus that reduces the decentralization of the system.

\section{- Practical Byzantine Fault Tolerance}

Practical byzantine fault tolerance (PBFT) is a consensus algorithm that was introduced in the late 90s [58]. It aims to improve the original Byzantine fault-tolerant algorithm. It was implemented in a popular blockchain platform named Hyperledger Fabric. It uses the PBFT which can handle almost one-third of malicious byzantine replicas [21,57]. Furthermore, this algorithm is gaining immense popularity because it can resist against software errors and malicious attacks. The main advantages of PBFT are that it consumes less energy because it does not carry out complex mathematical computations like PoW, and it's transactions do not require multiple confirmations. PBFT is a promising solution only when a small group of nodes is involved. However, it becomes inefficient in case of large networks. Also, PBFT is susceptible to Sybil attacks which is another weakness of the PBFT model.

\section{Recent Ongoing Project and a Use Case of Blockchain in CTs}

This section outlines and discusses the recent ongoing project and a use case of blockchain in CTs research. The purpose is to show how blockchain technology can revolutionize the current CT systems.

\section{A. The Triall Foundation's Verial Software Application ${ }^{1}$}

The Triall foundation has announced world's first CT in production on the blockchain. A beta version of this foundation's Verial software application has been given a green signal for use in CTs after assessing its compliance with international standards for medical research. It was used in a real-world CT (phase II, $<50$ participants) as a pilot project to optimize usability. The Triall foundation aims to ensure smarter, safer, and efficient clinical trial management. The primary focus of the foundation is to deal with the crucial challenges that make CTs operation complex, lengthy, and inefficient. These challenges usually arise in CTs due to the involvement of multiple organizations, locations, and data sources that hinder clinical researchers to keep a track record of their documents and data. The in-developed blockchain-based solution can help to prepare tamperproof documentation for all the conducted trials. The in-development application envisions revolutionizing CTs research and development by assuring the integrity and transparency of clinical evidence. Also, it aims to offer advanced solutions in terms of CTs data access. The in-development application promises to add significant values in CT procedures (e.g., informed consent, predefined outcome measures, and database lock). By registering a hash of the ICF on the blockchain can help researchers to prove that the patient has consented to

\footnotetext{
${ }^{1}$ Accessed on: 25 February 2020, https://medium.com/@niels.klomp/the-worlds-first-clinical-trial-in production-on-the-blockchain-has-just-been-announced-by-the-e05b73557dd0
} 
participate in CTs in a timely and verifiable manner. Similarly, registering a hash of the predefined plan on the blockchain can help researchers to prove whether or not they have achieved the predefined outcomes. To prove that CT data set has not been modified, can be done by generating a single hash of the entire CT database. In summary, Verial is a blockchain-based document management solution that has the potential to ensure integrity, authenticity, traceability, auditability, and immutability in CT research.

\section{B. Pharmaceutical Clinical Trials ${ }^{2}$}

Pharmaceutical CTs suffer from serious challenges, such as lack of trust between the multiple parties, complexity, patient recruitment, lack of regulatory standards, and data privacy and security. Throughout the CT procedure, multiple parties are involved which pose high costs, trust, and integrity issues. Blockchain is a potential technology that offers auditability and traceability features to pharma companies. Uploading data on blockchain helps to enhance transparency and trust as the stored data cannot be manipulated. Blockchain is based on consensus algorithms that help to get rid of the third party which was previously used to validate the transaction. The inherent features of blockchain technology can ensure data quality and provide a novel governance model in CTs research. Patient recruitment and traceability of data can also be assured using blockchain. The data of the patients involved in CTs is sensitive, and thus it does not make publicly available and easily accessible. On the other hand, due to multiple locations and centralized storage, it is become very challenging to trace back data or to recall that who accessed or modified the data at a specific interval of time. Blockchain can help to deal with such issues as it is based on distributed ledger systems that can be shared with third parties, such as CROs. This helps patients to keep full control of their data via public or permissioned blockchain. Throughout the CT, data remains immutable on blockchain because the same data is shared and stored on multiple systems, so an unauthorized change can be easily detected and verified. Blockchain can offer time-stamped data collection that leads to find verified identities that have entered in a CT system. In sum, blockchain is an emerging technology that can help in reshaping and transforming the current CT systems.

\section{Open Research Challenges}

The blockchain technology is in its infant stage with a central focus on improving the privacy and scalability factors [66]. The successful deployment and adoption of trustworthy blockchain systems in CTs requires additional research to solve numerous economic, organizational and technical challenges to make it a mainstream technology [67]. We briefly discuss several indispensable open challenges and issues in the following section.

\section{A. Scalability}

In certain CT scenarios, the blockchain becomes heavy due to the increasing number of transactions that occur daily, resulting in scalability issues. For instance, nearly 288,000 transactions occur per day or on average almost 3.3 transactions per second to compensate for the computation workload in a Bitcoin PoW model [44]. On the other hand, credit cards, such as Visa compute almost 150 million transactions per day or on average almost 2000 transactions per second [44]. Although the maximum theoretical number of transactions for Bitcoin is 7 transactions per second due to the block size limitation and the time needed to create a new block [20]. This becomes an issue when creating real-time blockchain-based solutions for healthcare applications because they will not be able to process millions of healthcare records in a real-time manner. Moreover, the block capacity is currently small, and thus minor transactions may be postponed as miners prefer to verify transactions that hold a high transaction reward; whereas, large size blocks slow down the transaction speed. Thus, scalability is a problematic issue in the current blockchain applications.

\section{B. Privacy leakage}

The blockchain networks must be safe enough because users generate addresses to make transactions instead of using their real identities. However, it has been reported that transactional privacy is not guaranteed in blockchain-based systems since the balances and transactions of each public key are made visible for each user in the network [22]. Recent studies reveal that users' information can be linked to their transactions. Efforts have been made to link user's IP addresses to their pseudonyms even when they are behind firewalls which are later on used to track the source of a particular transaction [20]. This problem is critical in the medical industry because

\footnotetext{
${ }^{2}$ Accessed on 25 February 2020, https://www.pharmafocusasia.com/strategy/blockchain-technologypharmaceutical-industry
} 
protecting patients' sensitive information is crucial. Also, further research needs to be conducted regarding the compliance of blockchain with GDPR, FDA, and other regulatory agencies as they ensure patients' privacy.

\section{Big Data Storage and low-cost adoption}

Blockchain enables decentralized storage for CTs data. Although decentralized storage mechanisms improve the storage performance in CTs, they pose numerous challenges with respect to high volume, variety, and velocity of data, also known as Big Data. Handling such a large amount of CTs data efficiently is very challenging. On the other hand, deployment of blockchain technology for clinical trials incurs a high cost which hinders its widespread adoption. To employ blockchain technology in clinical trials requires building new software which is very costly. It is estimated that software development costs around $\$ 10,000$ for basic applications to $\$ 800,000$, or more, for complex software. This high cost makes it unlikely to be adopted in clinical trials. Thus, substantial attention must be paid to resolve the higher cost issue.

\section{Selfish mining}

Blockchains are vulnerable to $51 \%$ attacks [44]. This generally occurs when malicious blocks are higher than honest blocks in a network. As a result, one of the possibilities is that a new block gets attached to the malicious chain as shown in figure 7. This strategy is called selfish mining because selfish miners keep their blocks private and reveal them to the public only when the private chain is longer than the current public chain, and thus it may be accepted by all miners in the network. While honest miners are wasting their resources on a meaningless branch, selfish miners are creating their private chain in the absence of competitors. Accordingly, selfish miners gain more revenue, as a result, rational miners are more attracted to join this community, thereby causing a greater chance of exceeding $51 \%$ of the power easily [20]. Thus, the nodes with over $51 \%$ of computing power can reverse the transactions in a blockchain. Hence, it poses serious security threats to CTs applications.

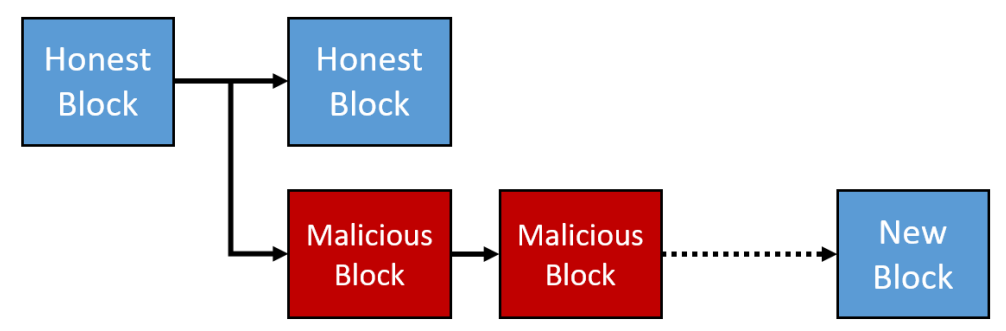

Figure 7: Selfish mining attack

\section{E. Cultural Shift and Data Disclosure Willingness}

Although digitalization is leading towards better insights and improved patients' outcomes in CTs, many CTs companies still rely on paperwork for certain processes. The immediate adoption of blockchain-based CTs would not be an easy task because changing people's behavior and altering their routine functions are very challenging for any industry, thereby high resistance from the employees is expected. Thus, the CTs companies need to figure out how to convince their employees in terms of a cultural shift. In this context, some novel incentive mechanisms need to be proposed. Also, adequate training must be provided to employees to implement blockchain technology. On the other hand, most of the hospitals do not agree on sharing their exact costing data with insurance companies because they usually charge differently to each patient. In such cases, where various stakeholders are not willing to share the exact information, implementing blockchain technology would be a crucial task.

\section{Conclusion}

This paper envisions to establish and expound how blockchain solutions can help to improve data integrity, patients' privacy and ensure CTs compliance with the regulatory policies. We presented a taxonomy that highlights the areas in CTs that can be enhanced using blockchain technology. The taxonomy was mainly divided into decentralized CT tasks and practices along with the blockchain types and its various consensus protocols. We provided insights on works in progress towards deploying blockchain solutions in CTs. Furthermore, we identified and discussed future challenges. Finally, we conclude that the extraordinary plethora of blockchain technology has provided remarkable support in healthcare by guaranteeing data provenance, 
transparency, decentralized transaction validation, and immutability. Blockchain technology proves to be beneficial in the pharmaceutical industry as it enforces data integrity through transparency, thereby reinforcing trust among all actors involved in a CT process. This technology not only leads to a community that is driven by trusted electronic health records but also bridges the gap between clinical researchers and patients. Although the distinctive features of blockchain help to improve clinical trial data management, the deployment of blockchain technology in CTs involves several indispensable challenges and issues that must be resolved.

\section{Acknowledgement}

This publication is based upon work supported by the Khalifa University of Science and Technology under Award No. CIRA-2019-001.

\section{References}

[1] S. Nakamoto, "Bitcoin: A Peer-to-Peer Electronic Cash System," [Online]. Available: https://bitcoin.org/bitcoin.pdf. [Accessed 4 Nov. 2019].

[2] S. Underwood, "Blockchain Beyond Technology," Communications of the ACM, vol. 59, no. 11, pp. 15-17, Nov. 2016.

[3] "Healthcare - Factom," Factom, [Online]. Available: https://www.factom.com/. [Accessed 5 Feb. 2019].

[4] "Healthnautica," [Online]. Available: https://www.healthnautica.com/comppages/index.asp. [Accessed 12 Aug. 2019].

[5] "Gem," [Online]. Available: https://gem.co/. [Accessed 24 Aug. 2019].

[6] "DNA.Bits," [Online]. Available: http://socialm1.wixsite.com/dnabits. [Accessed 19 Sep. 2019 ].

[7] "BlockVerify," [Online]. Available: http://www.blockverify.io/. [Accessed 21 Sep. 2019].

[8] M. Mettler, "Blockchain Technology in Healthcare: The Revolution Starts Here," in IEEE 18th International Conference on e-Health Networking, Applications and Services (Healthcom), Munich, Germany, 2016.

[9] Z. Shae and J. Tsai, "On the Design of a Blockchain Platform for Clinical," in IEEE 37th International Conference on Distributed Computing Systems, Atlanta, GA, USA, 2017.

[10] V. Anand, D. Scales, C. Parshuram and et.al, "Registration and design alterations of clinical trials in critical care: a cross-sectional," Intensive Care Med., p. 40(5): 700-22, 2014.

[11] N. Jones, "Half of US clinical trials go unpublished," Nature- Internatonal weekly journal of science, Dec. 2013. [Online]. Available: https://www.nature.com/news/half-of-us-clinical-trialsgo-unpublished-1.14286. [Accessed 22 April 2019].

[12] J. Brogan, I. Baskaran and N. Ramachandran, "Authenticating Health Activity Data Using Distributed Ledger Technologies," Computational and Structural Biotechnology Journal, 16 (2018): 257-266.

[13] B. Marr, "This Is Why Blockchains Will Transform Healthcare," Forbes, Nov. 2017. [Online]. Available: https://www.forbes.com/sites/bernardmarr/2017/11/29/this-is-why-blockchains-willtransform-healthcare/\#7ac6d8bd1ebe. [Accessed 13 March. 2019].

[14] A. Stanley, "Big Pharma Seeks DLT Solution for Drug Costs," coindesk, Jan 2018. [Online]. Available: https://www.coindesk.com/blockchain-day-big-pharma-seeks-dlt-solution-drug-costs. [Accessed 18 July. 2019].

[15] K. Sultan, U. Ruhi and R. Lakhani, "CONCEPTUALIZING BLOCKCHAINS:CHARACTERISTICS \& APPLICATIONS," in 11th IADIS International Conference Information Systems, Lisbon, Portugal, 2018. 
[16] J. Garay, A. Kiayias and N. Leonardos, "The Bitcoin Backbone Protocol: Analysis and Applications," Springer Berlin Heidelberg, pp. 281-310, 2015.

[17] A. Gervais, G. O. Karame, V. Capkun and S. Capkun, "Is bitcoin a decentralized currency?," IEEE Security Privacy, vol. 12, pp. 54-60, May 2014.

[18] I.-C. Lin and T.-C. Liao, "A Survey of Blockchain Security Issues and Challenges," International Journal of Network Security, vol. 19, no. 5, pp. 653-659, Sept. 2017.

[19] D. G. Glover and J. Hermans, "Improving the Traceability of the Clinical Trial Supply Chain," Applied Clinical Trials, pp. 36-38, Nov./Dec. 2017.

[20] E. Kiktenko, N. Pozhar, M. Anufriev, A. Trushechkin, R. Yunusov, Y. Kurochkin, A. Lvovsky and A. Fedorov, "Quantum-secured blockchain," Quantum Science and Technology, vol. 3, no. 3, pp. 1-6, June 2018.

[21] Z. Zheng, S. Xie, H.-N. Dai, X. Chen and H. Wang, "Blockchain challenges and opportunities: a survey," Int. J. Web and Grid Services, vol. 14, no. 4, p. 352-375, 2018.

[22] A. Kosba, A. Miller, E. Shi, Z. Wen and C. Papamanthou, "Hawk: The blockchain model of cryptography and privacy-preserving smart contracts," IEEE Symposium on Security and Privacy (SP'16), pp. 839-858, May 2016.

[23] H. Watanabe, S. Fujimura, A. Nakadaira, Y. Miyazaki, A. Akutsu and J. Kishigami, "Blockchain contract: Securing a blockchain applied to smart contracts," in IEEE International Conference on Consumer Electronics (ICCE'16), Las Vegas, NV, USA, 2016.

[24] J. Benet, "IPFS - Content Addressed, Versioned, P2P File System," 2014. [Online]. Available: https://arxiv.org/pdf/1407.3561.pdf. [Accessed 18 Dec. 2018].

[25] P. Labs, "Filecoin: A Decentralized Storage Network," July 2017. [Online]. Available: https://filecoin.io/filecoin.pdf. [Accessed 18 Dec. 2018].

[26] J. H. Hartman, I. Murdock and T. Spalink, "The Swarm Scalable Storage System," in 19th IEEE International Conference on Distributed Computing Systems, Austin, USA, 1999.

[27] R. Brooks, A. Skjellum, C. Worley, J. S. Obeid and L. Lenert, "Scrybe: A Blockchain Ledger for Clinical Trials," 2018 [Online]. Available: https://blockchain.ieee.org/images/files/images/clinicaltrialsforum2018/Clemson_WhitePaper.pdf. [Accessed 15 Dec 2018].

[28] J. Karlberg and M. Speers, "Reviewing Clinical Trials: A Guide for the Ethics Committee," China, March 2010. [Online]. Available: https://www.pfizer.com/files/research/research_clinical_trials/ethics_committee_guide.pdf. [Accessed 16 Dec. 2018].

[29] G. S. Borioli and J. Couturier, "How blockchain technology can improve the outcomes of clinical trials," British Journal of Healthcare Management, vol. 24, no. 3, pp. 156-162, March 2018.

[30] M. Benchoufi and P. Ravaud, "Blockchain technology for improving clinical research quality," BioMed Central, vol. 18, pp. 1-5, July 2017.

[31] S. Petersen and T. Hediger, "The Blockchain (R)evolution - How Blockchain technology can revolutionise the Life Sciences and Healthcare industry," Deloitte, 2017.

[32] "Patients Recruitment Forecast in Clinical Trials," Cognizant 20-20 insights, Aug. 2015. [Online]. Available: https://www.cognizant.com/whitepapers/patients-recruitment-forecast-in-clinicaltrials-codex1382.pdf. [Accessed 5 Jan 2019]. 
[33] C. Cai, "New rule on clinical trial reporting doesn’t go far enough," STAT, 17 Jan. 2017. [Online]. Available: https://www.statnews.com/2017/01/17/clinical-trial-reporting-new-rule/. [Accessed 5 Jan 2019].

[34] P. Schueler and B. Buckley, Re-Engineering Clinical Trials - Best Practices for Streamlining the Development Process, Academic Press, 2014.

[35] S. Jadhav, "Blockchain: The next big disruptor in clinical trials?," Pharmaceutical Engineering, vol. 38, no. 4, p. 25, July-Aug. 2018.

[36] M. Miliard, "IBM Watson, CDC on the hunt for new blockchain apps for healthcare," Healthcare IT News, 27 Oct. 2017. [Online]. Available: https://www.healthcareitnews.com/news/ibm-watsoncdc-hunt-new-blockchain-apps-healthcare. [Accessed 18 Jan. 2019].

[37] J. A. DiMasi, H. G. Grabowski and R. W. Hansen, "Innovation in the pharmaceutical industry: New estimates of r\&d cost," Journal of Health Economics, vol. 47, p. 20-33, 2016.

[38] F. Angeletti, I. Chatzigiannakis and A. Vitaletti, "Privacy preserving data management in recruiting participants for digital clinical trials," HumanSys'17, pp. 7-12, Nov. 2017.

[39] C. P. Gross, R. Mallory, A. Heiat and H. M. Krumholz, "Reporting the recruitment process in clinical trials: who are these patients and how did they get there?," Annals of Internal Medicine, vol. 137, pp. 10-16, 2002.

[40] M. C. Moken, "Fake pharmaceuticals: How they and relevant legislation or lack thereof contribute to consistently high and increasing drug prices," Am. $J L \&$ Med, vol. 29, p. 525, 2003.

[41] F. Angeletti, I. Chatzigiannakis and A. Vitaletti, "The role of blockchain and IoT in recruiting participants for digital clinical trials," in 25th International Conference on Software, Telecommunications and Computer Networks (SoftCOM), Split, Croatia, 2017.

[42] D. Bradbury, "The problem with Bitcoin," Computer Fraud \& Security, pp. 5-8, Nov. 2013.

[43] Gazali, S. Kaur and I. Singh, "Artificial intelligence based clinical data management systems: A review," Informatics in Medicine Unlocked, vol. 9, p. 219-229, 2017.

[44] T.-T. Kuo, H.-E. Kim and L. Ohno-Machado, "Blockchain distributed ledger technologies for biomedical and health care applications," Journal of the American Medical Informatics Association, vol. 24, no. 6, p. 1211-1220, Sept. 2017.

[45] "Gem Health Network," Gem, [Online]. Available: https://enterprise.gem.co/health/. [Accessed 5 Feb. 2019].

[46] "BitHealth | Devpost," BitHealth, [Online]. Available: https://devpost.com/software/bithealth. [Accessed 5 Feb. 2019].

[47] Y. B. Perez, "Medical Records Project Wins Top Prize at Blockchain Hackathon," CoinDesk, Nov. 2015. [Online]. Available: https://www.coindesk.com/medvault-wins-e5000-at-deloittesponsored-blockchain-hackathon. [Accessed 5 Feb. 2019].

[48] X. Yue, H. Wang, D. Jin, M. Li and W. Jiang, "Healthcare Data Gateways: Found Healthcare Intelligence on Blockchain with Novel Privacy Risk Control," Journal of Medical Systems, vol. 40, pp. 1-8, 2016.

[49] K. Safavi and B. Kalis, "Digital health tech vision 2018," Accenture, May 2018.

[50] R. Krawiec, D. Housman, M. Whit, M. Filipova and et.al, "Blockchain: Opportunities for Health Care," Deloitte, pp. 1-3, Aug. 2016.

[51] S. Angraal, H. M. Krumholz and W. L. Schulz, "Blockchain Technology Applications in Health Care," Circulation: Cardiovascular Quality and Outcomes-American Heart Association, pp. 1-3, 2017. 
[52] "Guardtime: Enterprise Blockchain," Guardtime, [Online]. Available: https://guardtime.com/. [Accessed 5 Feb. 2019].

[53] Z. Zheng, S. Xie, H. Dai, X. Chen and H. Wang, "An Overview of Blockchain Technology: Architecture, Consensus, and Future Trends," in IEEE 6th International Congress on Big Data, Honolulu, HI, USA, 2017.

[54] H. Journal, "Largest Healthcare Data Breaches of 2018," HIPAA Journal, Dec. 2018. [Online]. Available: https://www.hipaajournal.com/largest-healthcare-data-breaches-of-2018/. [Accessed 22 Jan. 2019].

[55] T. Nugent, D. Upton and M. Cimpoesu, "Improving data transparency in clinical trials using blockchain smart contracts," F1000Research, vol. 5, pp. 1-4, 2017.

[56] E. Androulaki, C. Cachin, C. Ferris, M. Sethi and et.al, "Hyperledger Fabric: A Distributed Operating System for Permissioned Blockchains," in EuroSys '18, Porto, 2018.

[57] T. T. A. Dinh, R. Liu, M. Zhang, G. Chen, B. C. Ooi and J. Wang, "Untangling Blockchain: A Data Processing View of Blockchain Systems,"IEEE Transactions on Knowledge and Data Engineering, pp. 2-7, 2017.

[58] M. Castro and B. Liskov, "Practical Byzantine Fault Tolerance," in Proceedings of the Third Symposium on Operating Systems Design and Implementation, New Orleans, 1999.

[59] G. Irving and J. Holden, "How blockchain-timestamped protocols could improve the trustworthiness of medical science," F1000research 5 (2016).

[60] A. Ramachandran and M. Kantarcioglu, "Using Blockchain and smart contracts for secure data provenance management," arXiv:1709.10000vl [cs.CR], 2017.

[61] S. Jiang, J. Cao, H. Wu, Y. Yang, M. Ma and J. He, "BlocHIE: a BLOCkchain-based platform for Healthcare Information Exchange," in IEEE International Conference on Smart Computing, Taormina, Italy, 2018.

[62] P. Zhang, JulesWhite, D. C. Schmidt, G. Lenz and S. T. Rosenbloom, "FHIRChain: Applying Blockchain to Securely and Scalably Share Clinical Data," Computational and Structural Biotechnology Journal, vol. 16, p. 267-278, 2018.

[63] O. Choudhury, N. Fairoza, I. Sylla and A. Das, "A Blockchain Framework for Managing and Monitoring Data in Multi-Site Clinical Trials," arXiv:1902.03975 [cs.DB], pp. 1-13, 2019.

[64] Y. Zhuang, L. Sheets, Z. Shae, J. J. P. Tsai and C.-R. Shyu, "Applying Blockchain Technology for Health Information Exchange and Persistent Monitoring for Clinical Trials," in AMIA Annual Symposium Poceedings 2018 (Vol. 2018, p. 1167).

[65] D. M. Maslove, J. Klein, K. Brohman and P. Martin, "Using Blockchain Technology to Manage Clinical Trials Data: A Proof-of-Concept Study," in JMIR Medical Informatics, 6.4 (2018): e1 1949.

[66] X. Xu, I. Weber, M. Staples, L. Zhu, J. Bosch, L. Bassz, C. Pautassox and P. Rimba, "A Taxonomy of Blockchain-Based Systems for Architecture Design," in IEEE International Conference on Software Architecture (ICSA), Gothenburg, Sweden, 2017.

[67] M. Staples, S. Chen, S. Falamaki, A. Ponomarev, P. Rimba, A. B. Tran, I. Weber, X. Xu and J. Zhu, "Risks and opportunities for systems using blockchain and smart contracts," in Data61 (CSIRO), Sydney, 2017. 